\section{Sicherheitstechnische und gesellschaftliche Aspekte von Monitoring bei der Endlagerung radioaktiver Abfälle mit Option ihrer Rückholbarkeit}

\author{
von Detlef Appel, PanGeo, und Jürgen \\ Kreusch, intac GmbH (beide Hannover)
}

\begin{abstract}
Die Endlagerung radioaktiver Abfälle in tiefen geologischen Formationen ist die bevorzugte Entsorgungsoption für hochradioaktive Abfälle. Die Umsetzung von Endlagerprojekten stößt jedoch oft auf erheblichen gesellschaftlichen Widerstand. Eine Ursache liegt in der Skepsis von Teilen der Bevölkerung gegenüber der passiven Gewährleistung der geforderten Langzeitsicherheit. Aktuelle Endlagerkonzepte sehen daher für einen gewissen Zeitraum, der für Monitoringmaßnahmen genutzt werden soll, die Rückholbarkeit von Abfällen vor. Die Monitoringergebnisse sollen die Sicherheitsaussagen absichern und so das Vertrauen der Bevölkerung in ein Endlager stärken. Zusammenhänge zwischen diesen Zielen sowie ihre Konsequenzen für Planung und Umsetzung von Monitoring und den Umgang mit den Ergebnissen werden diskutiert.
\end{abstract}

\section{Warum Rückholbarkeit und Monitoring?}

Bei der internationalen Fachdiskussion über die Entsorgung radioaktiver Abfälle aus der friedlichen Nutzung der Kernenergie hat sich - insbesondere für hochradioaktive Abfälle - deren Endlagerung in tiefen geologischen Formationen frühzeitig als bevorzugte Option herauskristallisiert. Dafür waren vor allem sicherheitstechnische und ökonomische Gründe ausschlaggebend. Obwohl diese Einschätzung im Wesentlichen bis heute Bestand hat, hat sich die Umsetzung dieser Entsorgungsoption in den meisten Ländern mit entsprechenden Endlagerprogrammen erheblich verzögert oder ist sogar bei der Auswahl eines Endlagerstandortes zunächst gescheitert. Ursachen dafür waren und sind Widerstände in der Gesellschaft gegen das Konzept passiv sicherer Endlagerung in tiefen geologischen Formationen bzw. - auch daraus resultierend - von Betroffenen gegen konkrete Endlagerpläne. Diese Haltung wird durch den Willen verstärkt, an gesellschaftlich relevanten Entscheidungen beteiligt zu werden.

Von vielen Menschen werden die unbestreitbaren, von den zuständigen Fachleuten als überwindbar eingeschätzten Unsicherheiten eines passiv sicheren, wartungsfreien Endlagersystems als bedeutsamer angesehen als die Unsicherheiten, die mit der Aufbewahrung der Abfälle in einem Lager mit aktiver Sicherheitsgewährleistung, also durch Überwachung und gegebenenfalls Reaktion auf unerwartetes Verhalten des Endlagersystems, verbunden sind. Nicht wenige Menschen halten sogar die überwachte „Dauerlagerung" der Abfälle in Bauwerken an der Erdoberfläche für die sicherste Entsorgungsstrategie. Die von vielen Fachleuten positiv beurteilte passive Sicherheit von Endlagern in tiefen geologischen Formationen stößt also in der Bevölkerung nicht durchwegs auf Zustimmung. Entsprechende Beurteilungsunterschiede bestehen auch gegenüber der Aussagekraft des Nachweises der Langzeitsicherheit für Endlagersysteme.

Die Argumente für oder gegen die passiv sichere Endlagerung einerseits und die Entsorgungsoptionen mit aktiver Sicherheitsgewährleistung andererseits werden seit langem kontrovers diskutiert (dazu z. B. Appel et al. 2001; EKRA 2000; ESK 2011). Auf Grund des anhaltenden Widerstands in der Gesellschaft wurde in einigen Ländern das ursprüngliche Endlagerkonzept - mit dem Ziel Vertrauensbildung - um die Rückholbarkeit von Abfällen ergänzt. Für die Betriebsphase eines Endlagers ist die Möglichkeit der Rückholung von Abfällen heute sogar Bestandteil der meisten nationalen Endlagerkonzepte für (insbesondere) hochradioaktive Abfälle.

Die Einführung der Rückholbarkeit von Abfällen aus einem Endlager allein leistet keinen Beitrag zur Reduzierung der endlagersystembzw. bewertungsimmanenten Unsicherheiten, die hinter der Forderung nach Modifizierung der passiv sicheren Endlagerung stehen; denn sie erlaubt allenfalls die Reaktion auf unvorhergese- 
hene Ereignisse bzw. Entwicklungen (deren Auftreten im Übrigen die sicherheitlichen Zweifel in der Bevölkerung bestätigen würde). Dies gilt insbesondere dann, wenn Rückholbarkeit auf die ohnehin vorgesehene Betriebsphase eines Endlagers beschränkt bleibt.

Andererseits ist eine über die normale Betriebszeit eines Endlagers hinausgehende Phase mit Rückholbarkeit der Abfälle aus dem wenigstens teilweise offenen Lager mit erheblichen Sicherheitsrisiken behaftet - etwa durch Aufrechterhaltung gebirgsmechanisch gestörter Verhältnisse, Austrag von Radionukliden mit Luft und Abwasser, radiologische Belastung der Beschäftigten und erleichterten Zugang zu spaltbarem Material. Die mögliche Vernachlässigung der erforderlichen Maßnahmen zur aktiven Sicherheitsgewährleistung als Folge gesellschaftlicher Veränderungen stellt einen weiteren Risikobereich dar (z. B. Appel et al. 2001). Seine Bedeutung ergibt sich insbesondere daraus, dass gesellschaftliche Systeme wegen ihrer großen Dynamik bereits für relativ kurze Zeiträume erheblich schwieriger zu prognostizieren sind als natürliche Endlagersysteme (s. dazu Buser 1998).

Eine Phase der Rückholbarkeit der Abfälle ist daher nur dann sinnvoll, wenn sie dazu genutzt wird, die Zweifel und Bewertungsunsicherheiten, die Anlass zu ihrer Einführung waren, durch gezielte Beobachtung des Endlagersystems zu reduzieren. Dabei ist allerdings sicherzustellen, dass die genannten Risiken reduziert bzw. kontrolliert werden. In diesem Sinne muss auch die Länge der Beobachtungsphase begrenzt und das Lager am Ende der Beobachtungsphase in den passiv sicheren Zustand überführt werden.

In einigen Ländern wird der Zeitraum der Rückholbarkeit von Abfällen und der Beobachtung über die normale Dauer der Betriebsphase eines herkömmlichen Endlagers voraussichtlich deutlich hinausgehen. Beispielsweise sollen in Frankreich die Rückholbarkeit von Abfällen und die Beobachtung des Lagers für mindestens 100 Jahre gewährleistet werden (ANDRA 2010). In der Schweiz ist die Beobachtungsphase als ,längerer Zeitraum, während dessen ein geologisches Tiefenlager vor dem Verschluss überwacht wird und die radioaktiven Abfälle ohne großen Aufwand zurückgeholt werden können“, definiert (KEG 2003, Art. 3). Ihre Dauer ist noch nicht festgelegt, bei der Konzeptentwicklung wurde von ,mehreren Jahrzehnten bis zu mehr als 100 Jahren" ausgegangen (EKRA 2002, S. 17).

Für die Phase der Rückholbarkeit und Beobachtung besteht die Notwendigkeit der aktiven Sicherheitsgewährleistung für das Endlager. Zugleich bietet sie die Chance, durch geeignete Untersuchungen die Aussagen zur Langzeitsicherheit eines Endlagers abzusichern und damit zur Entscheidungsfindung über das weitere Vorgehen (Beendigung der Beobachtung und Überführung des Endlagers in den passiv sicheren Zustand, Maßnahmen gegen kritische Entwicklungen des Endlagersystems) beizutragen. Nur für Untersuchungen mit dieser Zielsetzung wird hier der (an sich facettenreichere) Begriff Monitoring verwendet.

Auf welche Weise und mit welchen konkreten Zielsetzungen die über die „normale“ Betriebsphase eines Endlagers hinausreichenden Beobachtungsmaßnahmen umgesetzt werden und welche Funktion ihre Ergebnisse im Rahmen der Endlagerentwicklung und -bewertung haben können, wird derzeit im Rahmen nationaler Endlagerprojekte (z. B. Frankreich, Schweiz) und in internationalen Projekten (z. B. MoDeRn, s. dazu Harway/White 2011) diskutiert und untersucht.

Einige der sicherheitlichen Zusammenhänge zwischen den sicherheitstechnischen und gesellschaftlichen Zielen der Beobachtung, den Möglichkeiten und Grenzen der Erhebung aussagekräftiger Informationen und der möglichen Gefährdung der sicherheitlichen Ziele der passiven Endlagerung durch eine über die herkömmliche Betriebsphase hinausgehende Beobachtungsphase werden nachfolgend diskutiert. Dabei wird insbesondere auf die Beziehungen zwischen sicherheitstechnischen und gesellschaftlichen Aspekten des Monitorings für einzelne Zeitabschnitte des mit Endlagerentwicklung verbundenen Entscheidungsprozesses eingegangen. 


\section{Bezug von Rückholbarkeit und Monitoring zu Phasen der Endlagerentwicklung}

Bei Endlagerkonzepten ohne Rückholbarkeit der Abfälle bzw. mit Rückholbarkeit nur in der Betriebsphase lassen sich drei Phasen der Projektentwicklung und -umsetzung unterscheiden: Vorbetriebsphase, Betriebsphase und Nachbetriebsphase (z. B. ESK 2011; Blommaert 2010). Die für die Phasenabgrenzung ausschlaggebende Betriebsphase umfasst den Bau und Betrieb des Endlagers. Sie endet mit dem unmittelbar nach Einlagerung der Abfälle vorgesehenen Verschluss des Lagers.

Sind Rückholbarkeit und Monitoring über die „normale“ Dauer der Betriebsphase hinaus vorgesehen, wird die betreffende Zeitspanne als Beobachtungsphase o. ä. bezeichnet. Diese kann als eigenständige Phase zwischen Betriebs- und Nachbetriebsphase aufgefasst werden oder als Abschnitt der Betriebsphase (z. B. EKRA 2000; EKRA 2002) bzw. der Nachbetriebsphase (z. B. Blommaert 2010).

\subsection{Vorbetriebsphase}

Die Vorbetriebsphase umfasst diejenigen Aktivitäten, die der Errichtung eines Endlagers vorangehen. Hierzu gehören die Diskussion und Entwicklung des Endlagerkonzeptes (z. B. ohne oder mit Rückholbarkeit), die Entwicklung und Umsetzung des Standortauswahlverfahrens sowie die Festlegung und Genehmigung des Endlagerstandortes.

Vor der konkreten Umsetzung des Auswahlverfahrens muss im Zuge eines wissenschaftlichen und gesellschaftlichen Diskussionsprozesses festgelegt werden, ob bzw. unter welchen Voraussetzungen ein Monitoring für das geplante Endlager erwünscht oder gar notwendig ist und welche übergeordneten Ziele damit erreicht werden sollen. Im Verlauf des Verfahrens müssen dann mit zunehmendem Grad der Konkretisierung die technischen und gesellschaftlichen Anforderungen an das Monitoring und seine Ausgestaltung festgelegt werden.
Hohe Bedeutung kommt der institutionellen Absicherung des über Jahrzehnte andauernden wissenschaftlichen und gesellschaftlichen Prozesses hinsichtlich der durch Monitoring zu klärenden Fragen und der damit verbundenen Probleme zu. Ob die einmal getroffene Entscheidung für Monitoring auch langfristig auf gesellschaftliche Zustimmung stößt oder aber durch neue wissenschaftliche Erkenntnisse überholt wird, muss in der Vorbetriebsphase noch offen bleiben. Generell muss deshalb eine gewisse Flexibilität gewahrt sein, um als notwendig erkannte Änderungen im Endlagerkonzept, auch zum Monitoring, umsetzen zu können.

\subsection{Betriebsphase}

Die Betriebsphase umfasst den Bau des Endlagers, die Einlagerung der Abfälle sowie die Verfüllung und den Verschluss des Lagers. Für ihre Dauer werden mehrere Jahrzehnte veranschlagt.

Bei Entscheidung für Rückholbarkeit und Monitoring müssen in dieser Phase die zuvor nur dem Grundsatz nach getroffenen Festlegungen zu den Zielen des Monitorings in der Beobachtungsphase, zu seiner Umsetzung und zur Bewertung der Ergebnisse soweit verfeinert werden, dass das Monitoringprogramm systematisch geplant und seine Umsetzung vorbereitet werden kann. Auch müssen die technischen und organisatorischen Voraussetzungen für das Monitoring geschaffen werden. Danach wird die vollständige Revidierung der Entscheidung für Monitoring und ihrer Folgen zunehmend schwierig, weil dann z. B. in bereits verfüllten Lagerbereichen Teile der Messinfrastruktur vorhanden sind; sie können die Schwachstellen hinsichtlich der (Langzeit-)Sicherheit darstellen, aber nur mit störenden Eingriffen in das Endlagersystem zurück gewonnen werden.

Monitoring, das auf die Betriebsphase oder nur auf die Zeit der Abfalleinlagerung beschränkt bleibt (s. dazu Harvey/White 2011), wird hier nicht behandelt; denn die Ergebnisse können einerseits durch die betrieblichen Aktivitäten im Endlager beeinflusst werden und repräsentieren andererseits einen Systemzustand, zu dem wichtige Barrieren des Endlagersystems 
ihre volle Funktion noch nicht erreicht haben. Der Erkenntnisgewinn im Hinblick auf Langzeitsicherheit und der Beitrag zur Vertrauensbildung sind daher fraglich. Dies schließt Monitoringmaßnahmen allein in der Betriebsphase - etwa zur Gewährleistung der betrieblichen Sicherheit oder zur punktuellen Verbesserung des Systemverständnisses - nicht aus. Sinnvoll kann es sein, für die Beobachtungsphase vorgesehene Monitoringmaßnahmen bereits in der Betriebsphase zu beginnen, um unterschiedliche Zustände des Endlagersystems erfassen zu können.

\subsection{Beobachtungsphase}

Im Falle der Entscheidung für Rückholbarkeit der Abfälle über die „normale“ Betriebsphase hinaus beginnt nach dem (teilweisen) Verschluss des Endlagers die Beobachtungsphase. Spätestens jetzt sind die konkreten Ziele des Monitorings sowie klare Regeln zum Monitoringablauf und zur Struktur und Organisation des Vorgehens bei der Beurteilung der Ergebnisse und bei der darauf beruhenden Entscheidungsfindung zum weiteren Vorgehen festzulegen.

Durch geeignete Monitoringmaßnahmen sind Informationen über das Endlagersystem $\mathrm{zu}$ erheben, die eine abschließende Langzeitsicherheitsaussage und damit die Entscheidung für die Überführung des Endlagers in den passiv sicheren Zustand oder - im Extremfall - zur Rückholung der Abfälle begründen. Die dafür erforderliche Dauer der Beobachtung ist zunächst unbekannt. Da die Entscheidung für Monitoring gesellschaftlich und sicherheitstechnisch begründet ist, muss sich auch die Beendigung der Beobachtungsphase auf sicherheitstechnische Erkenntnisse und deren (auch) gesellschaftliche Bewertung stützen.

Seit Beginn der Vorbetriebsphase werden bis zum Beginn der Beobachtungsphase 50 bis 100 Jahre vergangen sein. Möglicherweise sind daher einige der Gründe für in diesen Phasen getroffene Festlegungen wegen gesellschaftlicher oder technisch-wissenschaftlicher Entwicklungen hinfällig. Das kann Konzeptänderungen bis hin zur Aufgabe des Monitorings erforderlich machen.
Monitoring ist also ein mehrere Phasen der Endlagerentwicklung übergreifender Vorgang.

\section{Umsetzung des Monitorings}

Nachfolgend werden einige Kernaspekte des Monitorings angesprochen, deren rechtzeitige und angemessene Beachtung für dessen Erfolg von erheblicher Bedeutung sind: Ziele des Monitorings, Messprogramm, Bewertung der gewonnenen Informationen und Einleitung resultierender Maßnahmen sowie verantwortliche Institutionen und Entscheidungsprozess.

\subsection{Ziele des Monitorings}

Das übergeordnete Ziel des Monitorings besteht darin, die Zweifel der Bevölkerung an der Langzeitsicherheit eines Endlagers bzw. der Zuverlässigkeit des entsprechenden Nachweises zu zerstreuen und die Akzeptanz gegenüber der Endlagerung bzw. einem bestimmten Endlager zu erhöhen.

Wenn mit Hilfe des Monitorings gezeigt werden kann, dass sich das Endlagersystem langfristig wie vorhergesagt entwickelt, kann daraus Vertrauen in den Sicherheitsnachweis erwachsen. Wichtiger für die Akzeptanz eines Endlagers ist aber die Möglichkeit, auf „ungünstige" Monitoringergebnisse mit Rückholung der Abfälle reagieren zu können. So gesehen ist die einvernehmliche Einführung einer Phase der Beobachtung des Endlagersystems der Kompromiss zwischen der von den gegenwärtig verantwortlichen Institutionen bevorzugten passiven Endlagerung und der von Teilen der Gesellschaft favorisierten aktiven Gewährleistung langzeitsicherer Entsorgung.

Aus den geschilderten Zusammenhängen lassen sich folgende sicherheitstechnische Hauptziele von Monitoring ableiten: Erkennen, Verstehen und Belegen der erwarteten Entwicklung und des langfristig stabilen $\mathrm{Zu}-$ stands des Endlagersystems als Voraussetzung für die Entscheidung zur Beendigung des Monitorings bzw. Erkennen und Verstehen einer unerwarteten Entwicklung als Voraussetzung für die Einleitung von Notfallmaßnahmen bis 
hin zur Abfallrückholung. Diesen Hauptzielen sind technische Nachweisziele zuzuordnen, bei deren Formulierung die aus den Eigenschaften des Endlagersystems und seiner erwarteten Entwicklung einerseits und der gesellschaftlichen Erwartungshaltung an das Monitoring sowie den technischen Möglichkeiten der Informationserhebung andererseits ableitbaren Anforderungen zu berücksichtigen sind.

\subsection{Messprogramm}

Umfang und Ausrichtung des Monitoringprogramms, also die zu wählende Instrumentierung, die Messgrößen, die Messorte und die Dauer des Programms hängen von den konkreten Monitoringzielen und den endlagerspezifischen Gegebenheiten ab. Dazu gehören vor allem die relevanten standortspezifischen Eigenschaften des Wirtsgesteins, das gewählte Endlagerkonzept und der Zeitraum, den das Monitoring (zunächst) umfassen soll. Da der genaue Zeitpunkt, $\mathrm{zu}$ dem die Monitoringziele erreicht sein werden, zunächst nicht bekannt ist, muss ein Prozess der fortwährenden Erfassung der festgelegten Parameter sowie der iterativen Bewertung der Ergebnisse und Entscheidung über das weitere Vorgehen etabliert werden. Lediglich die Dauer des ersten Iterationsschrittes kann und muss zeitlich festgelegt werden.

Das Messprogramm sollte so früh wie möglich während der Endlagerentwicklung beginnen, damit für die Bewertung eine möglichst umfassende Datenbasis zur Verfügung steht. In die Festlegung des Programms müssen Vertreter der Öffentlichkeit einbezogen werden, da nur so das erforderliche Vertrauen in das Messprogramm als Voraussetzung für das Mittragen der erzielten Ergebnisse erworben werden kann.

Beim derzeitigen Entwicklungsstand steht vor der erfolgreichen Umsetzung des Monitorings die Beantwortung einer Reihe von Fragen, die u. a. folgende Aspekte betreffen: Auswahl der zu messenden Parameter, räumliche und zeitbezogene Repräsentativität der Messwerte, Umgang mit Indikatoren für komplexe Vorgänge im Endlager, Integration mehrerer Parameter zu einer übergreifenden Funktionsaussage,
Funktionsfähigkeit und Zuverlässigkeit von Messinstrumenten, möglicher negativer Einfluss von Messeinrichtungen auf die Sicherheitsbarrieren, Dauer des Monitorings sowie Feststellung des die Langzeitsicherheit bestimmenden Systemzustands.

\subsection{Bewertung der gewonnenen Informationen und Einleitung resultierender Maßnahmen}

Die Bewertung der durch Monitoring gewonnenen Informationen und die darauf aufbauende Entscheidung über das weitere Vorgehen setzt die Entwicklung eines Bewertungs- und Maßnahmenplans voraus, und zwar vor Monitoringbeginn. Darin ist festzulegen, wie die Monitoringdaten bewertet werden und bei welchen Befunden bzw. Situationen welche Maßnahmen eingeleitet werden sollen oder müssen. Für den Fall der Abfallrückholung ist rechtzeitig zu klären, wo diese zwischengelagert werden sollen.

Bei der Erstellung und Umsetzung dieses Monitoringplans muss die Öffentlichkeit umfassend und wirksam beteiligt werden.

Für die Datenbewertung müssen parameterspezifische Bewertungsmaßstäbe und -regeln abgeleitet werden, damit anhand der gewonnenen Messwerte einvernehmlich entschieden werden kann, ob sich das Endlagersystem wie erwartet entwickelt oder ob Abweichungen auftreten.

Der Berücksichtigung dieser einfachen Notwendigkeiten stehen wissenschaftliche und methodische Probleme entgegen, die im Vorfeld eines Monitoringprogramms gelöst werden müssen oder für die eine Umgangsweise festzulegen ist. Sie stehen beispielsweise mit folgenden Fragen in Zusammenhang: Auf Grundlage welcher Kenntnisse werden die Bewertungsmaßstäbe abgeleitet? In welcher räumlichen und zeitlichen „Dichte“ müssen die Messwerte anfallen, um ausreichend genau die Entwicklung des Endlagersystems darstellen zu können? Wie sollen Gruppen oder die Gesamtheit der Messwerte zu einem Gesamtergebnis aggregiert werden? Wie sind fehlerhafte von korrekten Messwerten zu unterscheiden? 


\subsection{Entscheidungsprozess und verant- wortliche Institutionen}

Die Umsetzung der durch das Monitoring erarbeiteten Erkenntnisse in Entscheidungen zum weiteren Vorgehen muss im Rahmen eines vorab vereinbarten Entscheidungsprozesses mit einvernehmlich festgelegten Regeln erfolgen.

Die Aufgaben und Verantwortungen der an diesem Prozess beteiligten Institutionen müssen klar geregelt und allen Beteiligten, einschließlich der Öffentlichkeit, bekannt sein. Die Verteilung ergibt sich aus den Antworten auf die Fragen „Wer misst?“, „Wer bewertet?“, „Wer trifft Entscheidungen?".

Wegen der weitreichenden sicherheitlichen und gesellschaftlichen Konsequenzen der zu treffenden Entscheidungen muss die fachliche Bewertung der Monitoringergebnisse (zumindest ihre Bestätigung) durch eine Institution (bzw. ein Gremium) erfolgen, die in ihren Entscheidungen von den verfahrensverantwortlichen Institutionen (insbesondere Betreiber, Aufsichts- und Genehmigungsbehörde) unabhängig ist. Sie muss durch Fachleute des Vertrauens der beteiligten Institutionen und Interessengruppen gebildet werden. Wegen des möglicherweise langen Zeitraums bis zur abschließenden Entscheidung über den Verschluss des Endlagers ist große Sorgfalt auf die Aufrechterhaltung der Arbeitsfähigkeit, des Problembewusstseins und der Fachkompetenz der Institution bzw. des Gremiums zu verwenden. Die Bewertungsergebnisse müssen der Öffentlichkeit zeitnah und vollständig vorgelegt werden. Resultierende Entscheidungen sind unter Beteiligung der Öffentlichkeit zu diskutieren und mit gesellschaftlicher Beteiligung zu treffen.

\section{Schlussfolgerungen}

Monitoring bietet die abstrakte Chance, das fehlende Vertrauen von Teilen der Bevölkerung in die passiv sichere Endlagerung radioaktiver Abfälle in tiefen geologischen Formationen und in den entsprechenden Langzeitsicherheitsnachweis für ein Endlager zu stärken bzw. zu gewinnen. Voraussetzung dafür ist aber, dass im
Rahmen der durch Monitoring ergänzten Endlagerung mit Rückholbarkeit von Abfällen bei entsprechenden Monitoringergebnissen und ihrer Bewertung Maßnahmen gegen sicherheitskritische Entwicklungen des Endlagersystems bis hin zur Rückholung der Abfälle möglich sind.

Das methodische Problem des Monitorings, zuverlässige Daten mit räumlich und zeitlich hinreichender Aussagekraft zum Systemverhalten und ohne nachteilige Konsequenzen für die Langzeitsicherheit eines Endlagers zu erheben, ist derzeit nicht abschließend gelöst. Entsprechendes gilt für die mit der Bewertung der erhobenen Befunde zusammenhängenden Probleme. Derzeit ist offen, wie zuverlässig die Entwicklung des Endlagersystems über einen langen Zeitraum durch ein Monitoringprogramm gemessen und beobachtet werden kann und wie auf dieser Basis sichere Aussagen zur Langzeitsicherheit bzw. Entscheidungen (bis hin zur Rückholung von Abfällen) getroffen werden können.

Wenn solchen Aussagen und Entscheidungen die Überzeugungskraft fehlt, wird das erhoffte Vertrauen nicht oder nur eingeschränkt zu gewinnen sein. Denn die Monitoringergebnisse und etwa darauf gegründete mögliche Konsequenzen werden dann die von Skeptikern angeführten Unsicherheiten der Endlagerung nicht ausgleichen können. Monitoring stellt deshalb per se keine Lösung der Streitfrage passiv sichere Endlagerung oder aktive Sicherheitsgewährleistung dar.

Monitoring kann im Sinne eines vertrauensbildenden Vorgehens nur dann erfolgreich sein, wenn die Gesellschaft, auch ihr endlagerungsskeptischer Anteil, von Beginn der Endlagerentwicklung an wirksame Beteiligungsmöglichkeiten (Mitsprache- und Entscheidungsrecht) besitzt. Vertrauensbildung in der Öffentlichkeit kann nur erreicht werden, wenn nicht nur naturwissenschaftlich-technische, sondern auch gesellschaftliche Gesichtspunkte in die Diskussion und Entscheidungsfindung einfließen. Sie müssen durch Repräsentanten von der Gesellschaft selbst eingebracht werden.

Unabhängig von den Aspekten Rückholbarkeit und Monitoring ist eine Lösung der Endlagerfrage nur dann zu erreichen, wenn alle Phasen 
der Endlagerentwicklung von einer intensiven gesellschaftlichen Diskussion begleitet werden und die Gesellschaft an den zugehörigen Entscheidungen beteiligt ist. Es ist daher frühzeitig - möglichst vor Beginn der Auswahl eines Endlagerstandortes - eine gesellschaftliche Grundsatzdiskussion über Entsorgungs- und insbesondere Endlagerungsoptionen in Gang zu setzen. Darauf aufbauend ist ein Verfahren zur Standortauswahl und Endlagerentwicklung mit Mitsprache- und Entscheidungsrecht der Bevölkerung zu erarbeiten und umzusetzen. Dabei ist rechtzeitig auch die Rolle, die Monitoring als Beitrag zur Vertrauensbildung leisten kann, zu klären. Spätes „Aufpfropfen“ von Monitoring zwecks Akzeptanzgewinns würde genauso scheitern wie die technokratisch entwickelten und umgesetzten Endlagerprojekte der Vergangenheit.

\section{Literatur}

ANDRA - Agence nationale pour la gestion des déchets radioactifs, 2010: The French Approach to Reversibility in Radioactive Waste Management. In: Aparicio, L. (ed.): Making Nuclear Waste Governable - Deep Underground Disposal and the Challenge of Reversibility; S. 29-70 http://www.andra.fr/download/andra-international-en/document/editions/381va.pdf (download 17.12.12)

Appel, D.; Kreusch, J.; Neumann, W., 2001: Vergleichende Bewertung von Entsorgungsoptionen für radioaktive Abfälle - Abschlussbericht. Hannover, im Auftrag des Forschungszentrums Karlsruhe, Projektträger des $\mathrm{BMBF}$ und $\mathrm{BMWi}$ für Wassertechnologie und Entsorgung; http://edok01.tib.uni-hannover.de/ edoks/e01fb01/334702852l.pdf (download 17.12.12)

Blommaert, $W$., 2010: Reflections on Flexibility, Reversibility, Retrievability and Recoverability by the Belgian Nuclear Safety Authority. Vortrag auf der R\&R-Tagung der NEA, Reims, 14.-17. Dezember 2010, FANC, Belgien

Buser, M., 1998: „Hüte“-Konzept versus Endlagerung radioaktiver Abfälle: Argumente, Diskurse und Ausblick. Gutachten zuhanden Hauptabteilung für die Sicherheit der Kernanlagen

EKRA - Expertengruppe Entsorgungskonzepte für radioaktive Abfälle, 2000: Entsorgungskonzepte für radioaktive Abfälle - Schlussbericht. Bern, im Auftrag des Departements für Umwelt, Verkehr, Energie und Kommunikation (UVEK); http:// www.bfe.admin.ch/php/modules/publikationen/ stream.php?extlang $=$ de \&name $=$ de_182456219. pdf\&endung $=$ Entsorgungskonzepte $\% 20 \mathrm{f} \% \mathrm{FCr} \% 20$ radioaktive $\% 20 \mathrm{Abf} \% \mathrm{E} 41 \mathrm{e}, \% 20 \mathrm{Schlussbericht}$ (download 17.12.12)

EKRA - Expertengruppe Entsorgungskonzepte für radioaktive Abfälle, 2002: Beitrag zur Entsorgungsstrategie für die radioaktiven Abfälle in der Schweiz. Bern, im Auftrag des Departements für Umwelt, Verkehr, Energie und Kommunikation (UVEK); http://www.bfe.admin.ch/php/modules/publikationen/stream.php? extlang $=$ de $\&$ name $=$ de 5031486 . pdf\&endung=Beitrag\%20zur\%20Entsorgungsstrategie $\% 20 \mathrm{f} \% \mathrm{FCr} \% 20 \mathrm{die} \% 20$ radioaktiven $\% 20$ Abf\%E4lle\%20in\%20der\%20Schweiz (download 17.12.12)

ESK - Entsorgungskommission, Ausschuss Endlagerung radioaktiver Abfälle (EL), 2011: Rückholung/ Rückholbarkeit hochradioaktiver Abfälle aus einem Endlager - ein Diskussionspapier; http://www.entsorgungskommission.de/downloads/epanlage2el19homepage.pdf (download 17.12.12)

Harway, L.; White, M., 2011: MoDeRn - Monitoring Developments for Safe Repository Operation and Staged Closure - Expert Stakeholders Workshop Report, 23.09.2011; http://www.modern-fp7.eu/filead$\mathrm{min} /$ modern/docs/Deliverables/MoDeRn_D5.3.1 Expert_Stakeholders_Workshop_Report.pdf (download 17.12.12)

KEG - Kernenergiegesetz der Schweiz, 2003: Kernenergiegesetz (KEG) vom 21. März 2003; http:// www.admin.ch/ch/d/as/2004/4719.pdf (download 17.12.12)

\section{Kontakt}

Dr. Detlef Appel

PanGeo - Geowissenschaftliches Büro

Ibykusweg 23, 30629 Hannover

E-Mail: appel-pangeo@t-online.de 